\title{
Alfabetización audiovisual en la escuela: un estudio de caso
}

Jesús Ramé López - Universidad Rey Juan Carlos

(D) 0000-0002-7480-949X

Recepción: 25.07.2021 | Aceptado: 16.10.2021

Correspondencia a través de ORCID: Jesús Ramé López

iD 0000-0002-7480-949X

Citar: Ramé López, J (2021). Alfabetización audiovisual en la escuela: un estudio de caso. REIDOCREA, 10(31), 1-15.

Área o categoría del conocimiento: Educación Mediática y Cultura Visual

Resumen: Estamos viviendo un momento de cambio de paradigmas comunicacional a través de la tecnología, el cual hace emerger una sociedad en la que se nos bombardea continuamente con mensajes audiovisuales. En consecuencia, existe un consenso entre asociaciones de familias e instituciones educativas, artísticas y políticas en relación a la necesidad de una alfabetización audiovisual. Se anuncian programas de implementación del audiovisual en la escuela y aparecen estudios en relación a este medio, pero no cristalizan en proyectos concretos, sino que se queda en la voluntariedad de los docentes, los cuales no se siente preparados ni acaban de encontrar el espacio del audiovisual en el currículo. Desde este punto de partida, nos hemos centrado en el estudio de caso de un centro en el que sí se da la materialización de un proyecto concreto. Dicho proyecto, cuyo nombre es "Educar la mirada", se realiza en Trabenco, un colegio público de Leganés, con un carácter general de centro, en horario lectivo y con un contenido curricular. Nuestra investigación pretende descifrar cuales son las claves para que tenga lugar esta iniciativa, siendo la alfabetización audiovisual una actividad que brilla por su ausencia en la escuela pública.

Palabra clave: Alfabetización Audiovisual

\section{Audiovisual literacy at school: a case study}

Abstract: We are living a moment of change in communicational paradigms through technology, which makes a society emerge in which we are continuously bombarded with audiovisual messages. Consequently, there is a consensus between family associations and educational, artistic and political institutions regarding the need for audiovisual literacy. Programs for the implementation of audiovisuals in schools are announced and studies appear in relation to this medium, but they do not crystallize into concrete projects, but rather the voluntary nature of the teachers, who do not feel prepared or have just found the space of the audiovisual in the curriculum. From this starting point, we have focused on the case study of a center in which the materialization of a specific project does occur. This project, whose name is "Educar la gaze", is carried out in Trabenco, a public school in Leganés, with a general character of a center, during school hours and with a curricular content. Our research aims to decipher which are the keys for this initiative to take place, audiovisual literacy being an activity that is conspicuous by its absence in public schools.

Keyword: Audiovisual Literacy

\section{Introducción}

El final del siglo XX y el inicio del XXI ha desplegado una revolución tecnológica sin precedentes en relación al audiovisual. En ningún momento de la historia hemos asistido a un bombardeo tan grande de imágenes (Berger, 2000). Tampoco habíamos asistido a tan masiva puesta en juego de dispositivos de grabación, postproducción y exhibición en el ámbito cotidiano. El móvil o internet han provocado que el lenguaje audiovisual se haya convertido en algo que rodea nuestras vidas. De este modo, han ido apareciendo categorías como el prosumidor (productor-consumidor) (Toffler, 1980) o EmiRec (Cloutier, 2001), que reflejan, en palabras de Mario Kaplún (1998), una relación entre los nuevos usos de de las TIC y nuevos modos pedagógicos, en un camino hacia la educomunicación (Aparici y García, 2017). Desde estos planteamientos teóricos queremos, también, descubrir cuales son los cambios en la escuela y que conflictos provoca esta transformación que están haciendo emerger los nuevos paradigmas comunicacionales, los cuales implican un uso del audiovisual más cotidiano e incluso 
necesario. No podemos tampoco perder de vista el planteamiento que introduce la alfabetización audiovisual (junto a la informacional, tecnológica y mediática) dentro de las nuevas alfabetizaciones, con lo que conlleva de nuevos aprendizajes.

Sin embargo, pese a que forma parte de nuestras rutinas, tanto como el lenguaje escrito u oral, no está en las aulas de forma tan natural como en el día a día de la vida de los educandos. Así, un estudio de 2015 del Gabinete de Comunicación y Educación de la Universidad Autónoma de Barcelona sobre el uso del audiovisual en las aulas españolas planteaba que "si la imagen y el audiovisual se incorporan a la enseñanza, cambian no solo el modo de ver y analizar el mundo, sino de estudiarlo, de transmitirlo, de aprender sobre él y de interactuar con la realidad y con los demás" (Gabinete de comunicación y educación de la Universidad Autónoma de Barcelona, 2015, p. 6). Lo cual no significa que los docentes manifestaran en el mismo informe su inseguridad ante el uso pedagógico del audiovisual en el aula.

Cada vez se escucha más que el audiovisual, como escribe Lukács sobre el cine, "tiene un «lenguaje» particular que es necesario «aprender» si se quiere tener acceso vivencial a las obras" (Lukács, 1967, p. 130) en este medio. Para las instituciones educativas parece ser que este acceso debería ser "consciente y fundamental" en la escuela, como declaraba, el 21 de septiembre de 2013, el ministro de Educación Cultura y Deportes José Ignacio Wert, cuando anunciaba la importancia para el gobierno de "la introducción en el sistema educativo de la mirada audiovisual, de la cultura audiovisual" (COPE, 2013). El anuncio se acompañaba de una medida concreta: la introducción de una asignatura tanto en Secundaria como en el Bachillerato. Este hecho parecería el inicio del ingreso del lenguaje audiovisual en las instituciones de aprendizaje españolas, pero la realidad de 2021 es que en la LOMCE (Ley Orgánica para la Mejora de la Calidad Educativa) la alfabetización audiovisual brilla por su ausencia, dejándose su implementación a la motivación o buena voluntad de los docentes que buscan un encaje de la transversalidad de este aprendizaje.

Esta investigación se ha desarrollado antes de la implantación de la LOMLOE (Ley Orgánica 3/2020), por lo tanto, las referencias legales se refieren a LOMCE (Ley Orgánica 8/2013). Más adelante, haremos un breve comentario sobre los cambios que pueden afectar a este proyecto con la LOMLOE. El encaje en la LOMCE de la alfabetización Audiovisual vendría dado desde las competencias (Real Decreto 126/2014, art. 2.2), que tienen un cambio respecto de la LOE (Ley Orgánica de Educación). En las competencias actuales no existe una propia que defina los estándares que debería alcanzar el educando competente en lenguaje audiovisual, estaría diseminada de forma transversal entre todas $y$, a juicio de los propios docentes (Gabinete de comunicación y Educación de la Universidad Autónoma de Barcelona, 2015). De hecho, la única vez que se nombra la "comunicación audiovisual" en el espacio de las disposiciones generales del desarrollo de competencias, que corresponde a la Orden ECD/65/2015, es en la competencia básica de comunicación lingüística: "Desde la oralidad y la escritura hasta las formas más sofisticadas de comunicación audiovisual o mediada por la tecnología, el individuo participa de un complejo entramado de posibilidades comunicativas gracias a las cuales expande su competencia y su capacidad de interacción con otros individuos" (Orden ECD/65/2015, LOMCE).

El interés institucional y de la sociedad en España por la alfabetización audiovisual, parece que es más grande, de hecho, existen propuestas con la firme intención de generar prácticas educativas que se integren en nuestros sistemas de aprendizaje. El ICAA (Instituto de la Cinematografía y de las Artes Audiovisuales) ha propuesto unos premios de alfabetización audiovisual en la escuela (Orden ECD/2657, 2015) y , además, puso a disposición de todas las personas que precisaran de información sobre 
estos proyectos, una base de datos con los proyectos más interesantes ${ }^{1}$. Incluso el Plan Estratégico General 2012-2015 de la Secretaría de Estado de Cultura definía como su estrategia 17 "Desarrollar políticas encaminadas a mejorar la recepción, comprensión y valoración por la ciudadanía de las obras y contenidos cinematográficos y audiovisuales en tanto que medios contemporáneos de comunicación social y expresión artística" (Secretaría de Estado de Cultura, 2012), además el desarrollo de dicha estratégica hacía hincapié en la importancia de la formación de un pensamiento crítico hacia los medios audiovisuales para la infancia y la adolescencia.

Además, la Academia de las Artes y las Ciencias Cinematográficas de España ${ }^{2}$, en colaboración con otros organismos y organizaciones, está impulsando la idea de que la enseñanza del lenguaje audiovisual tiene que ser una parte fundamental del aprendizaje institucional, haciendo incidencia en el cine como arte. Esta institución ha creado ya, en colaboración con el Ministerio de Educación, Cultura y Deporte, un portal educativo de cine español (MECD, 2017). La "Academia" plantea además que, a través del Plan Cultura 2020 (MECD, 2017), el gobierno cree un programa de Alfabetización Audiovisual dentro del proyecto "Educación para el Cine. Cine para la Educación". Además, esto se hace más urgente cuando la mayoría de los docentes siguen pensando que no ven un encaje claro de la alfabetización audiovisual en el currículo actual (Gabinete de comunicación y Educación de la Universidad Autónoma de Barcelona, 2015).

¿Cuál es el motivo de que no cristalicen estos proyectos educativos en la escuela pública? En resumen, la preocupación social existe, hay un intento de impulsar medidas desde las instituciones y parece también que la comunidad educativa está interesada en el audiovisual desde sus diferentes actores (docentes, familias y educandos). Entonces, ¿Por qué existen tan pocos ejemplos de proyectos de alfabetización en la escuela, y mucho menos en centros públicos en horario lectivo?

Si escuchamos a los docentes, éstos manifiestan en un 69,4\% que es importante o muy importante la falta de formación en la escuela española para la implantación de una alfabetización audiovisual (Gabinete de comunicación y Educación de la Universidad Autónoma de Barcelona, 2015), aunque existe un Plan de Cultura Digital en la Escuela de 2013 impulsado por el Ministerio de Educación, Cultura y Deporte. En el Marco Común de Competencia Digital del Docente (INTEF, 2017) se desarrollan las competencias audiovisuales que deberían tener los docentes, que son desde el manejo de vídeos para sus clases hasta el fomento de la creación audiovisual y el manejo de algunos dispositivos. Esto choca con el sentimiento de preparación de los docentes y con su práctica cotidiana, ya que sólo el 33,2 \% de los docentes declara hacer un uso frecuente del audiovisual en sus acciones pedagógicas. Podría parecer que el problema viene también de la falta de recursos, pero el $78 \%$ considera que la infraestructura de sus escuelas es buena o muy buena (Gabinete de comunicación y Educación de la Universidad Autónoma de Barcelona, 2015). Así el uso del audiovisual en la escuela se convierte en algo voluntarioso más que con un marco pedagógico a aplicar, dificultando el desarrollo de un proyecto de alfabetización común. En otro sentido, pero no menos importante, los docentes no consideran en su mayoría que el audiovisual forme parte de una práctica común en la escuela. En consecuencia, el $83 \%$ considera que la alfabetización audiovisual no forma parte del currículo obligatorio, el 93\% lo define como un contenido transversal y el sólo $16 \%$ de los docentes de primaria ve el estudio del audiovisual como una materia que se imparte como contenido relacionado (Gabinete de comunicación y Educación de la Universidad Autónoma de Barcelona, 2015).

\footnotetext{
${ }^{1}$ https://www.mecd.gob.es/cultura-mecd/areas-cultura/cine/promocion/educacion-audiovisual/proyectosalfbetizacion.htm

${ }^{2}$ La Academia ha publica un monográfico sobre el tema en la revista $A C A D E M I A$, además ha impulsa unas guías didácticas para docentes.
} 
Por todo esto, nuestra investigación toma valor en función de una aparente incapacidad para implementar este aprendizaje en el sistema educativo, haciéndose necesarias las respuestas a por qué sucede. Nosotros vamos a partir de la búsqueda de algún lugar donde sí se ha hecho posible la implementación de la alfabetización audiovisual, dejando para una próxima investigación la factibilidad de estos proyectos en otras escuelas.

Así, nos sorprende el caso de Trabenco, un colegio público de Leganés (Madrid) el cual lleva 10 años realizando un proyecto de alfabetización audiovisual llamado "Educar la Mirada" (Trabenco, 2018). Dicho proyecto se organiza en horario lectivo, a través de talleres semanales de hora y media, y es parte del desarrollo curricular de los educandos. Ha sido premiado con menciones de honor en dos de las ediciones del concurso de alfabetización audiovisual del ICAA y forma parte de la base de datos, que esta misma institución tienen con los proyectos de alfabetización audiovisual más destacados de España. Aquí surge el interrogante que resume nuestra investigación: ¿Por qué en esta escuela pública es posible la implementación curricular de un proyecto de alfabetización audiovisual?

No pretendemos, en ningún caso, hacer una investigación que apoye la idea de que los colegios públicos tengan que ser como nuestro objeto de estudio. Nuestra idea es sacar los elementos que hacen emerger este proyecto y describir el contexto que lo permite, de tal forma que podamos entender lo que facilita procesos concretos, para valorar finalmente la posibilidad de una alfabetización audiovisual en la escuela. Desde este planteamiento surgen diferentes interrogantes y estrategias para resolverlos: ¿Por qué en este colegio se puede dar este proyecto?

\section{Objetivos e hipótesis}

Todos los objetivos que planteamos a continuación organizan el desarrollo de nuestra investigación. Los hemos colocado antes de la metodología para que se entiendan algunas de las intenciones de ésta.

El objetivo general de esta investigación es conocer cuál puede ser el desarrollo curricular de la alfabetización audiovisual en la escuela. En consecuencia, al tener este análisis un carácter de trabajo de campo, tenemos los siguientes objetivos específicos

1. Descubrir las condiciones del centro y de la comunidad educativa (Trabenco), para que se pueda producir el desarrollo de un proyecto como "Educar la Mirada".

2. Ver el funcionamiento de los diferentes paradigmas comunicacionales en la enseñanza.

3. Sacar a la luz herramientas y didácticas fértiles para la implementación de los dispositivos audiovisuales en el aprendizaje.

4. Explicar el encaje de los talleres y del proyecto de alfabetización audiovisual en Trabenco dentro de la LOMCE.

Así, si en la mayoría de los colegios públicos no son capaces de generar algún tipo de acción pedagógica que integre el aprendizaje del lenguaje audiovisual, quizá sea por el proyecto educativo y dependa de los diferentes agentes y sus disposiciones de compromiso para con la comunidad educativa en dicho proyecto. En consecuencia, podemos deducir que el cambio educomunicacional no sea un tema electoralmente rentable para llevar a cabo por las diferentes administraciones políticas, ya que implicaría muchos cambios, en la estructura económica y organizativa de la educación. Mucha complejidad a largo plazo y, aparentemente, poca rentabilidad electoral. Esta hipótesis se ampara en la observación de que el colegio Trabenco tiene unas 
características concretas que permiten el desarrollo de experiencias educativas, que en otras escuelas sonarían a desconocidas, cuando no imposibles de realizar ${ }^{3}$.

Como dice Marco Polo en Las ciudades invisibles de Italo Calvino: "Si quieres saber cuánta oscuridad tienes alrededor, has de aguzar la mirada para ver las débiles luces lejanas" (Calvino, 2015, p. 73). Esto pretendemos con nuestra investigación, dar cuenta de un proyecto que ha dado sus frutos en la alfabetización audiovisual, con el fin de definir las prácticas educativas puestas en juego y entender por qué no se da en otros proyectos educativos.

\section{Método}

Nuestra investigación se define como un estudio de caso, ya que nos centraremos en una acción pedagógica dentro de un centro educativo concreto: El proyecto de alfabetización audiovisual ("Educar la mirada") en el colegio público Trabenco. Además, tiene un carácter antropológico en el sentido de que nuestra observación del proyecto ha comenzado como un estudio de campo ya antes del inicio de la investigación concreta sobre la que vamos a hacer hincapié. De hecho, daremos a la investigación un carácter descriptivo y no propositivo, en un intento de que los resultados sean científicos, desde un sentido fenoménico.

El objeto de estudio (La comunidad educativa del colegio público de Leganés Trabenco) es complejo, ya que incluye al claustro, a las familias, los educandos, un modo de relación propio y un vínculo institucional con la administración tanto autonómica como estatal. Esto implica que tengamos que utilizar varias herramientas de investigación, y que nos obligue a ir cambiando de una a otra conforme avance el análisis. Para la organización de un marco metodológico general Utilizaremos el pensamiento modal, viendo el objeto de estudio como un modo de relación. Para ello partiremos de la ontología de Hartmann (1956) y del desarrollo para el análisis de los modos de relación de Jordi Claramonte (2016). Estas herramientas epistemológicas, las aplicaremos desde una perspectiva de la Filosofía de la educación. Así, desde el pensamiento modal Avanzaremos en detectar los repertorios - la categoría que explica una colección de formas coherente- que permiten la realización del proyecto "Educar la mirada", donde aparecerán los modos de lo necesario y de lo contingente. También tendremos la categoría de las disposiciones - las inteligencias y actitudes que pone en juego el objeto de estudio para cumplirse de forma autónoma y coherente-, donde los modos de 10 posible y lo imposible definen la emergencia de una forma de hacer. Finalmente, en este hacerse cargo de sus proyectos de los colectivos humanos, sus modos de hacer se cristalizarán en la categoría de complexo, que es lo que emerge como tal, es decir, en nuestro caso de estudio, el proyecto de alfabetización audiovisual. Dentro de esta categoría aparecerían los modos de lo efectivo y de lo inefectivo, que son esos atractores que al final equilibran lo que se va decantando como lo que finalmente emerge. Con estos parámetros pondremos en juego nuestra observación del proyecto, en ese carácter antropológico que hemos señalado.

Nos parece también muy oportuno el análisis de documentos que explicitan la necesidad de una alfabetización audiovisual, pero también los documentos que el centro público Trabenco debe desarrollar para mantener sus prácticas educativas dentro de los estándares de aprendizaje dictados por la Comunidad de Madrid y el Estado español.

\footnotetext{
${ }^{3}$ El colegio público Trabenco, como explicaremos en el desarrollo de la investigación, es un centro de línea 1 (una clase por curso), lo que pertenece a una modalidad de escuela que está en continuo retroceso. En este colegio, por ejemplo, el método de evaluación es cualitativo y no cuantitativo, además de que las familias en su conjunto, aparte de pertenecer todas al AMPA, toman decisiones colectivas como la de no recoger las notas para no fomentar la competitividad entre sus hijos e hijas.
} 
Un ejemplo claro es la PGA (Plan General Anual), donde debe estar todo el planteamiento práctico que se va a desarrollar durante el curso, a lo que se suma la explicación del encaje educativo de acciones pedagógicas como los talleres o la definición de la participación de las familias en el centro educativo. Otro documento muy importante para análisis son el RRI (Reglamento de Régimen Interno) y el PEC (Proyecto Educativo del Centro), que tienen todos los centros públicos y que define la línea educativa, sus actuaciones pedagógicas y la organización del centro. La reflexión sobre estos documentos nos dará una visión de conjunto en la cual incorporar los resultados del análisis del proyecto de alfabetización audiovisual.

En el carácter cualitativo de nuestra investigación se hace conveniente, no sólo saber la opinión de los protagonistas de este proyecto de alfabetización audiovisual —familias (AMPA), claustro (Docentes y equipo directivo) Y educandos-, sino también expertos y representantes de las organizaciones educativas como la FAPA.

\section{Resultados}

El colegio público Trabenco está ubicado en Leganés, población periférica de Madrid, y en este año cumple sus 48 años. Es un colegio de línea 1 (una clase por curso) y lo integran los tres cursos de infantil y los seis cursos de primaria, con lo cual tienen 9 clases. Las ratios de la comunidad de Madrid mantienen las clases en 25, salvo casos ocasionales de adaptación de los centros a situaciones especiales que la comunidad puede obligar a ser 30 (BOE, 2012). El colegio tiene un proyecto educativo donde la participación de familias es fundamental y donde la organización asamblearia es la base de la toma de decisiones (Trabenco, 2021). Se organizan por comisiones de trabajo que forman docentes y familias. Sus 6 principios de identidad son: libre expresión y creatividad, actitud crítica responsabilidad y solidaridad, valoración de la diversidad, aprender investigando y gestión democrática y participativa (Trabenco, 2021) ${ }^{4}$.

En su método de evaluación no se utilizan ni exámenes, ni boletines de notas ${ }^{5}$. Tienen evaluación continua a través de la observación directa del alumno/a en su actividad individual o grupal y en distintos contextos: diario de clase tanto del alumnado como del docente, registro de observación, entrevistas personales con alumnado y familias, asambleas de clase, de familias y generales.

El proyecto "Educar la Mirada" (Trabenco, 2018) surge, como nos explica Marisa Víctor (antigua profesora y una de las personas que pone en marcha la iniciativa), de la inquietud de las familias y docentes de $2^{\circ}$ ciclo, las cuales iniciaron un debate sobre si el audiovisual educa. Del debate salieron muchas conclusiones, pero una evidente era que quedaba claro que audiovisual educa, aunque no sabían ni cómo ni en qué sentido. Lo interesante del inicio del proyecto es que nace del debate dentro de la comunidad educativa y que se resuelve en una acción pedagógica concreta, ya que deciden iniciar un taller de alfabetización audiovisual, intentando involucrar a familias y a docentes para generar una investigación y experimentación sobre la comunicación audiovisual. La pretensión era intentar que los educandos profundizaran en el medio y que pudieran reconocer las estrategias técnicas y creativas para tener un conocimiento pleno de su lenguaje. En este sentido, tanto Marisa Víctor como Mariano García (presidente del AMPA), nos han manifestado que al igual que en otros lenguajes, como el teatro, el alumnado era capaz de interpretar y hacer una crítica de obras artísticas, con el

\footnotetext{
${ }^{4}$ Debemos añadir que el centro tiene jornada continua y acaba su horario lectivo a las 16:30, lo cual provoca que la participación de familias sea más fácil. En el proyecto de alfabetización audiovisual es el espacio de mayor desarrollo, ya que los talleres se desarrollan por la tarde. ${ }^{5}$ Como comentamos antes, existe un acuerdo entre familias de no recoger los boletines obligatorios de evaluación cuantitativa.jeplo
} 
audiovisual quedaban fascinados y no iban mucho más allá de si les gustaba o no lo que visionaban. Con estos intereses comienza el taller de alfabetización audiovisual que se suma a otros talleres y que se dirige a $2^{\circ}$ y $3^{\circ}$ ciclo. Este se realiza con grupos interciclos mezclados de los cuatro cursos $\left(3^{\circ}, 4^{\circ}, 5^{\circ}\right.$ y $\left.6^{\circ}\right)$ y en las tardes de los miércoles. Este es el germen, que luego cristalizará en una idea de proyecto de centro que se desarrollará hasta nuestros días, el cual ha ido incorporando dinámicas y herramientas educativas en función de lo que iban descubriendo en el desarrollo de las experiencias que iban realizando. Cómo figura en el proyecto educativo, se desprende una preocupación por la sociedad de las pantallas y por las herramientas de análisis, lo cual les hace insistir en tres aspectos: actitud crítica, conocimiento de las alternativas y creatividad.

Otra herramienta es el "cine en familia", que pretende acercar otras alternativas audiovisuales a las familias desde la reflexión en relación a una pieza audiovisual concreta como "El globo rojo" (Albert Lamorisse, 1956). El proyecto explica que:

"es una apuesta por buscar espacios en familia, preparados y cuidados, en los que pasar buenos ratos con esa magia que solo el cine consigue, estar juntos, conversar... Queda muy lejos ya de este uso habitual de la televisión como aparato permanentemente encendido que nos va sirviendo en bandeja y sin esfuerzo lo que ella decide y nosotros tragamos sin apenas digestiones" (Trabenco, 2018).

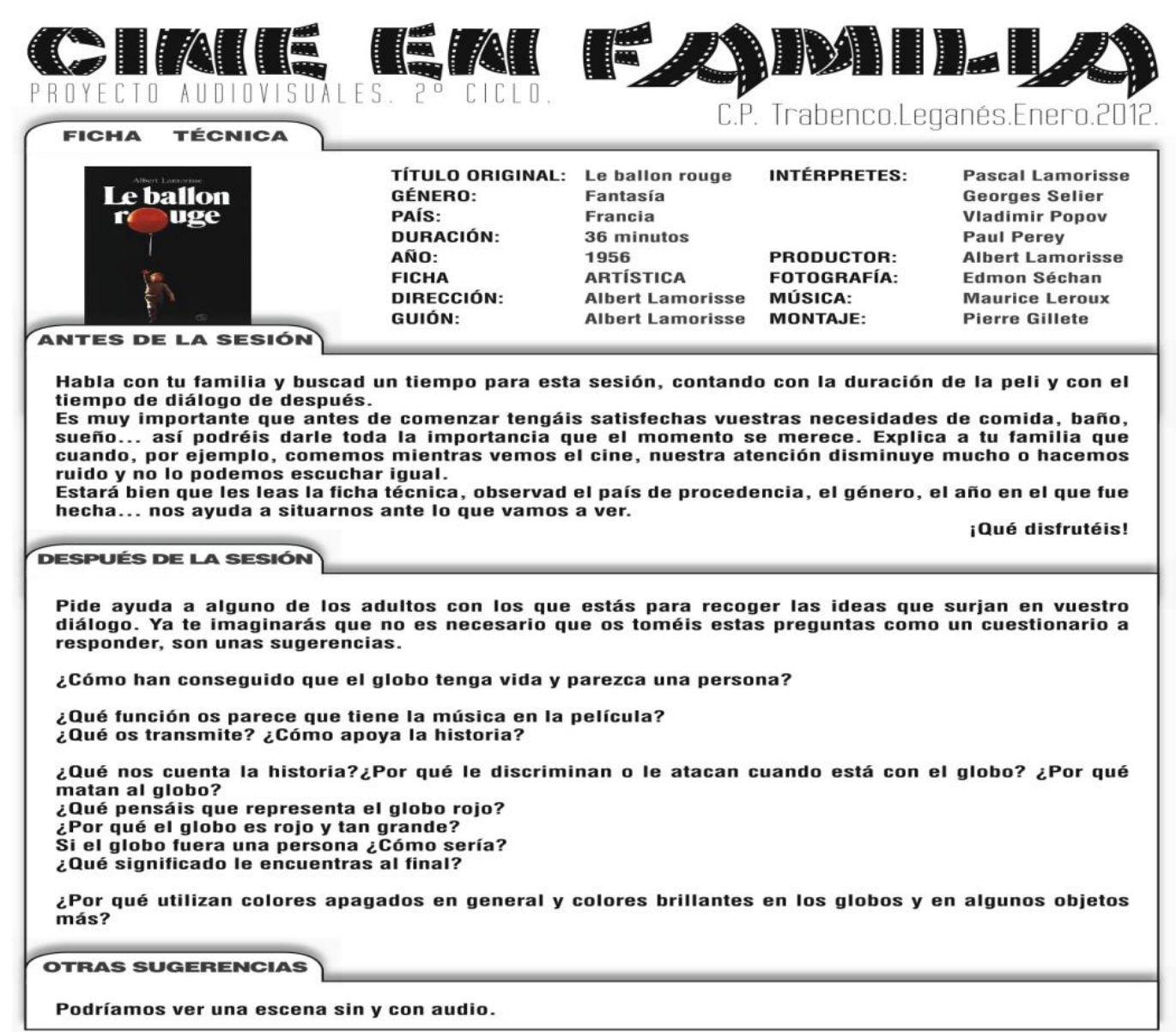

Figura 1. Cartel de Cine en familia, explicación de una sesión de Cine en Familia en el colegio Trabenco; fotografiado del archivo del AMPA del colegio Trabenco (2012).

Esta herramienta permita la reflexión en familia de forma activa, donde la actitud crítica se convierte en una actividad gratificante que se vive y se disfruta. Otra de las 
herramientas muy interesante es lo que el proyecto denomina "Cine inteligente". Esta actividad como explican en su proyecto:

"Pretende ofrecer cada cierto tiempo en el colegio, la posibilidad de compartir una 0 varias piezas audiovisuales, disfrutando de un posterior diálogo entre personas de edades muy diferentes pues este es un reto importante como comunidad educativa, necesitamos espacios comunes que nos encuentren, que nos enseñen a escucharnos, en los que divertirnos juntas, más allá de planteamientos de actividades en las que las personas adultas vamos como acompañantes de la infancia, o actividades en las que el público infantil parece que no tuviera cabida" (Trabenco, 2018).

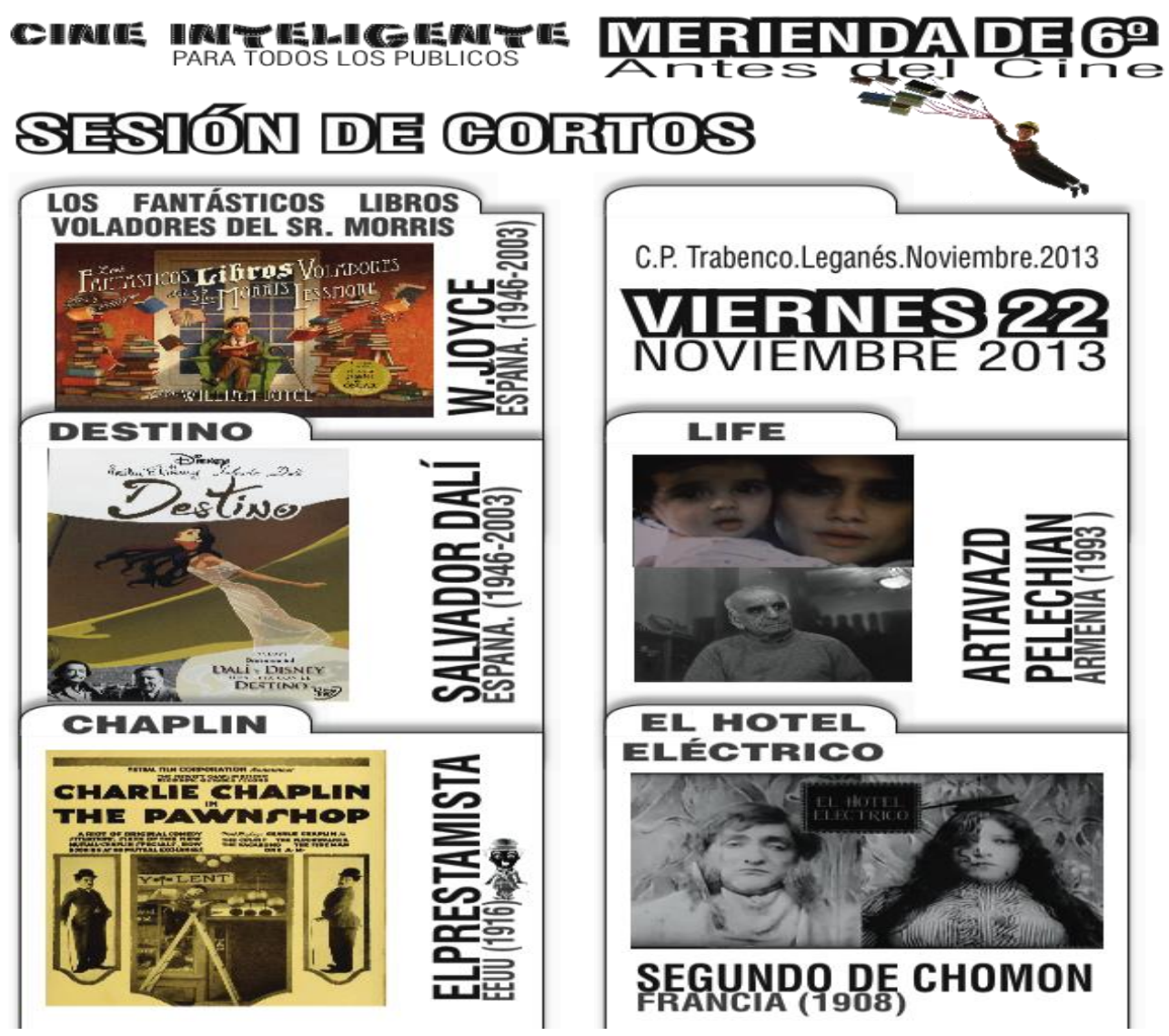

Figura 2. Cartel de Cine Inteligente, Programación de una sesión de Cine Inteligente en el colegio Trabenco; fotografiado del archivo del AMPA del colegio Trabenco (2013).

Esta actividad también se complementa con el visionado de las propias creaciones del proyecto, lo cual permite también reflexionar colectivamente sobre las obras realizadas en el curso lectivo en el que se hayan realizado.

Al mismo tiempo todo esto se combina con el uso del audiovisual cuando hay un intercambio (actividad que se da en 6을 de primaria), ya que permite la mediación de un sistema de comunicación más allá del lenguaje oral. El stop motion se convierte, en este caso, no solo en una herramienta de comunicación sino para la comunicación. La idea de la intersubjetividad se complementa tanto con encuentros con profesionales como las salidas, experiencias que hacen el aprendizaje vivencial. La herramienta última que han sumado es una maleta de cine para hacerla como tarea voluntaria en casa, lo cual se relaciona con la idea de un proyecto global de centro, que tiene coherencia con lo que viven las familias en sus hogares.

Los talleres son la herramienta pedagógica fundamental con la que se organiza el proyecto de alfabetización audiovisual "Educar la mirada" y son una parte fundamental 
de los procesos pedagógicos expresados en el proyecto educativo. En el proyecto que dio pie al premio Marta Mata del ministerio de educación al centro, escriben que los "talleres permiten realizar actividades de manera más diversificada y trabajar en la comunidad educativa (alumnado, profesorado y familias) el respeto a zonas compartidas y materiales comunes" (Trabenco, 2009). Se realizan en todas las etapas del centro menos en primero de infantil, ya que al ser una tarde a la semana este curso todavía mantiene la siesta. El centro comienzo el horario lectivo a las 9:30 y termina a las 16:30, con lo cual tienen jornada continua. Intentan mantener grupos pequeños (alrededor de 12 educandos por taller) que pasen por diferentes talleres en el año. Además, estos grupos son heterogéneos y organizados con el criterio del claustro, donde se mezclan edades, sexo, cultura, motivación, estilo de aprendizaje, inteligencias, capacidades, Esto implica que los talleres estén organizados para la diversidad que requiere la complejidad educativa del centro. Se programan por áreas de conocimiento y por el desarrollo de diferentes lenguajes.

Tienen separados los talleres en inter-ciclos por ritmos de aprendizajes e intereses, de tal forma que los cursos de 4 y 5 años de infantil se juntan con los del primer ciclo de primaria, quedando el segundo y tercer ciclo de primaria unidos en grupos para la organización de dichos talleres. A todo esto, se debe añadir que los talleres son organizados por docentes y familias, repartiéndose los cometidos, ya que la base didáctica y la relación con el currículo es parte de las maestras y maestro, siendo las familias las que ponen los conocimientos y habilidades en las materias a tratar y los recursos humanos necesarios para la consecución del taller.

Como nos lee Víctor González, profesor de infantil, desde el ejemplo del Plan General Anual del curso 2010-2011, los planteamientos de los talleres que se han mantenido durante los últimos años en el centro responden a las preguntas de ¿Por qué?, ¿Para qué? y el ¿Cómo?:
"¿por qué? Las propuestas de talleres suponen el desarrollo de estrategias de trabajo individual y en grupo. Se sustenta en la participación activa, el intercambio y la construcción colectiva del conocimiento en u clima de respeto y de interacción ¿Para qué? Las estrategias a desarrollar se fundamentan en el trabajo cooperativo, reconocer que la construcción conjunta supera los aportes individuales porque los integra ¿Cómo? Las actividades se desarrollan mediante técnicas que permiten actuar alrededor de la tarea a realizar" - y añade - "pero todas las herramientas y didácticas tienen que tener un sentido dentro de aprender a aprender y aprender para la vida" (Trabenco, 2010).

Como el taller de audiovisuales sustenta el proyecto de alfabetización, este necesita de un desarrollo didáctico pensado por los docentes, el cual implique que la acción pedagógica responda a una situación de aprendizaje. Las características que deben cumplir son la participación activa, que exista un intercambio de pensamientos, que haya una construcción colectiva del conocimiento, que se experimente con materiales y herramientas, que exista apoyo mutuo, cooperación y ayuda entre iguales. La intención es que los saberes sean compartidos por el alumnado con diferentes adultos de la comunidad educativa y, si procede, con personas ajenas a la comunidad educativa que puedan aportar algún aspecto deseado al proyecto. En esto entra la visita de profesionales o las salidas.

El taller de audiovisuales cumple claramente los criterios generales que se pretenden con esta herramienta pedagógica: permite educar para la vida y en la vida, ya que el audiovisual forma parte del quehacer cotidiano del día a día del alumnado; es un aprendizaje funcional, muy motivador para los educandos y con sentido para éstos; aprenden experimentando, haciendo e interactuando con sus iguales; con el uso del diálogo, haciéndose preguntas e hipótesis que se van desvelando en el proceso de aprendizaje. 
Mariano García nos cuenta que en el proyecto de alfabetización audiovisual han intentado llevar a cabo los principales objetivos de los talleres:

- Fomentar la iniciativa personal y ajustarla a la grupal, potenciando las capacidades de curiosidad, espontaneidad y creatividad.

- Estimular la imaginación, la exploración y la auto expresión.

- Favorecer el intercambio y la cooperación en una tarea grupal.

- Desarrollar la capacidad de observación y reproducción, ajustando la propia acción a la finalidad consensuada.

- Experimentar con diferentes formas de comunicación y representación utilizando distintas técnicas y utensilios.

El centro cuenta, además, con recursos audiovisuales como cámaras, trípodes, proyectores, ordenadores..., los cuales se ponen a disposición del taller. Además, el centro o el AMPA se hacen cargo de recursos que sean necesarios en alguna actividad puntual como salidas a un museo. Hay que destacar que el proyecto se complementa con el "cine en familia", el "cine inteligente" y tareas voluntarias como la maleta viajera que tienen que ser programadas por el docente tutor de cada clase y ayudado por las familias encargadas del proyecto de alfabetización audiovisual.

Los talleres forman parte de la planificación anual del centro y del proyecto educativo, por lo tanto, el desarrollo de estos son parte fundamental del quehacer educativo del centro. Así, el proyecto de alfabetización audiovisual está introducido desde el mismo planteamiento general del centro desde las áreas de conocimiento que implica y su lenguaje. El audiovisual en su análisis y práctica es atravesado por todas las competencias básicas de la LOMCE (Ley Orgánica 3/2020), lo cual permite un desarrollo de estos aspectos de la ley, aunque al mismo tiempo, al no estar encuadrada en una concreta, implica un gran esfuerzo pedagógico de planificación. Al inicio de curso se hace una justificación en la cual aparecen los horarios, pero además en los objetivos generales de la PGA ya están marcados los fines de las situaciones de aprendizaje. Dentro del desarrollo de las áreas de conocimiento en el proyecto curricular aparecen planificaciones por ciclos o inter-ciclos, que además aterrizan en la programación de aula que tiene cada docente, el cual vuelve a programar por situaciones de aprendizaje desarrollando los objetivos de aprendizaje que se plantean en la LOMCE. Todo esto requiere una gran coordinación de la comunidad educativa que tiene más fácil despliegue en un colegio de línea 1. Así se hacen elementos fundamentales la evaluación continua y la introducción de contenidos: por ejemplo, al escribir el plan de rodaje de un haiku audiovisual y su respectivo poema, se están trabajando conceptos de lengua y literatura, pero además se está desarrollando la competencia lingüística. Al ser la observación y la coevaluación de los trabajos la base de la valoración del proyecto permite la puesta en juego de una alfabetización audiovisual efectiva, para la vida no para un examen.

Debemos explicar en este momento algunos cambios de LOMLOE que no modifican los planteamientos anteriores, pero que le dan cierto amparo institucional desde la ley. En esta nueva ley, que ha entrado en vigor para el curso 2021/22, se refleja un interés transversal, tanto en el preámbulo como en los objetivos y desarrollo de las materias en primaria en secundaria obligatoria. La ley repite, en diferentes ocasiones (Artículos 17, 19, 24 y 25), la necesidad de que las diferentes asignaturas en su desarrollo se hagan cargo de la alfabetización audiovisual. En el preámbulo de la LOMLOE se expresa claramente que:

"Se deberá dedicar un tiempo diario a la lectura y se establece que la comprensión lectora, la expresión oral y escrita, la creación artística, la comunicación audiovisual, la competencia digital, el fomento de la creatividad y del espíritu científico se trabajarán en todas las áreas de educación primaria" (Ley Orgánica 3/2020, 2020, p. 122873). 
Este mismo párrafo se expresa para la educación secundaria obligatoria, dejando un claro hueco para la introducción en los contenidos de aprendizaje, siendo a través de una organización de las programaciones pedagógica, las cuales podrían resultar en un desarrollo de talleres dentro de un ajuste curricular.

Otro ejemplo del proyecto de alfabetización es el uso en educación infantil y primaria de los pensamientos de la práctica denominada "pedagogía de los F.P.R." (Bergala, 2007). Los fragmentos de películas generan un desplazamiento de significado que también provoca la aparición de ideas propias sobre la obra por parte del espectador. Ha sido muy fértil contemplar la experiencia de diferentes directores o creadores audiovisuales cuando mostraron sus secuencias favoritas a los chicos y chicas de la escuela. Este uso del fragmento se complementaría con la creación de una dvdeteca, que tratara al cine como arte, además de hacer que el cine sea una herramienta de conocimiento en el aula, como sucede con los libros. "No se trata de formar otro gusto sino simplemente de formar un gusto" (...) "Que el cine entre en los hábitos por impregnación" (Bergala, 2007).

Debemos destacar la fertilidad de los talleres en relación al cumplimiento de los objetivos educativos, ya que se convierten en una compensación de los objetivos de las áreas de conocimiento. Por eso los talleres son susceptibles de cambios cada año, de tal forma que se adapten a necesidades concretas (curriculares, de diversidad...). A esto podemos sumar que pueden recoger intereses sociales como, por poner un ejemplo actual, el feminismo. El audiovisual tiene la capacidad de poder incluir desde contenidos hasta temas de actualidad, por eso se convierte en un medio que, en este caso a través de un proyecto de centro, genera cultura de escuela, donde los educandos pueden tener un sentido de pertenencia a un espacio de relaciones que tiene unos fines y necesidades propias que desarrollar y en el que toda la comunidad educativa está incluida.

Nos hemos encontrado con una comunidad educativa muy cohesionada, en la cual el trabajo en equipo es real y cada acción pedagógica que se contempla tiene un número de personas y recursos que permiten un desarrollo satisfactorio de cada actividad que plantea el proyecto de alfabetización audiovisual. Los horarios son concretos las tardes de los miércoles y jueves, lo cual, como nos comenta Belén Cruz (Miembro del AMPA), permite a las familias la planificación su vida para poder participar. Además, esas tardes el colegio se pone a disposición de los talleres, con lo cual los espacios y recursos están a la entera disposición de los participantes de los talleres.

Las familias, lejos de entender la colaboración como un engorro, muestran entusiasmo por su participación y se implican. Un ejemplo reciente es el de la maleta de cine, que en el año pasado desarrolló una película colectiva con planos de diferentes horas del día. La experiencia está resultando muy fértil e incluso los adultos están mandando sus planos, aparte de los propios que han hecho sus hijos e hijas. La organización asamblearia del centro también facilita que se esté informado y que se pueda actuar colectivamente sobre cualquier aspecto del proyecto.

El carácter asambleario de la comunidad educativa Trabenco, la relación entre familias y claustro es muy estrecha, ya que las decisiones se toman colectivamente. Además, los canales de comunicación son múltiples: la relación directa que permite el clima de familiaridad del centro, donde las familias pueden incluso acompañar a sus hijos e hijas hasta el aula. A esto sumamos las agendas que aparecen en primaria, las asambleas y juntas de toda la comunidad educativa (que son más o menos cada mes), las asambleas de ciclo, las asambleas de aula y las entrevistas personales entre familias y docentes. Con este planteamiento se requieren familias y claustros comprometidos con el desarrollo de la educación, de tal forma que se está cultivando continuamente una cultura de escuela y la creación de una comunidad educativa fuerte. Esta relación de la comunidad educativa está reflejada en el proyecto educativo. 
Una de las claves por la que siendo una escuela pública y en la que el proyecto se desarrolla en horario lectivo es que es un colegio pequeño. Al ser de línea 1 (una clase por curso) solo existen nueve clases en todo el centro, de tal forma que las relaciones que se producen son más intensas y ampliadas a todos los miembros de la comunidad educativa. Hemos observado que los educandos se conocen independiente de su edad, y esto se debe tanto a el pequeño número de alumnos y alumnas como a que tienen muchas oportunidades de vivir actividades en común. Es muy común ver cómo, en nuestras visitas al centro, las familias se saludan por el patio, por los mismos motivos que se crean los modos de relación del alumnado.

El centro además está abierto a propuestas de visitas de profesionales y a hacer salidas que tengan relación con lo tratado en el proyecto de alfabetización audiovisual. Esto facilita su elaboración, ya que, por ejemplo, cuando estaban trabajando los trucos Méliès, coincidió con una exposición del cineasta en Caixa Fórum y el claustro determinó que era una salida necesaria para cumplimentar el taller. Lo mismo ocurrió con la visita a una exposición sobre el surrealismo, cuando se trató este tema en el proyecto.

Quizá uno de los aspectos más interesantes del proyecto de alfabetización audiovisual sea como afecta a las familias esta acción educativa. Nos hemos dado cuenta de que el proyecto en este sentido se desarrolla en varios aspectos, ya que hay diferentes niveles de participación. Por un lado, las familias forman parte del taller, por lo cual tanto los participantes con conocimientos audiovisuales como los que no, se enriquecen de forma natural con el encuentro con la infancia y con el desarrollo del taller. Además, durante la realización del "cine inteligente" y el "cine en familia", se adoptan nuevas formas de hacer que ponen en juego nuevos modos de relación con el audiovisual. La maleta del cine que se ha puesto en marcha este año también pone de manifiesto ese cambio de relación con el medio, ya que tienen que crear planos que deben organizarse dentro de la familia para justarse a los horarios y condiciones de creatividad.

Nos parece que esta relación de las familias con los medios se acerca a cambios en los modos de ver, a cambios en los paradigmas, tan necesarios en nuestra sociedad multipantalla, que en este caso se producen de forma natural. La creación de una reflexión y debate en familia permite la emergencia de un espacio para la actitud crítica, lugar donde podemos generar herramientas de análisis colectivo.

\section{Discusión y conclusiones}

Nos hemos dado cuenta de que el proyecto "Educar la mirada" parte de una idea del audiovisual como una representación, un reflejo, en palabras de Buckingham (2005), "versiones selectivas" del mundo. Esto provoca que, en muchos otros casos, proyectos de alfabetización audiovisual se planteen con una idea de aprendizaje similar al de la literatura, como una asignatura concreta en los planes educativos, pero al mismo tiempo la investigación educativa actual se cuestiona la categoría de asignatura. En estos momentos del debate pedagógico nos encontramos con un cambio de paradigma educacional que de manera inevitable afecta al estudio de la introducción del audiovisual. Así, en Trabenco parten de una definición de alfabetización audiovisual que se dirigiría hacia un aprendizaje de los conocimientos, prácticas y competencias para comprender y hacer uso de su medio. Aunque sabemos que tomar el audiovisual análogamente con el lenguaje es algo impreciso, pensamos que a este proyecto le hace entender su propia propuesta de reflexión sobre el sistema de comunicación audiovisual. Lo que sí nos interesa destacar de la idea de alfabetización audiovisual, que mantienen en el centro, es su carácter crítico, porque, como bien explican, no es únicamente importante entender su mecanismo, también es necesario reflexionar sobre sus efectos. 
Parece evidente que existe una preocupación social por los mensajes audiovisuales, esto nos lleva a pensar en lo más importante de este proyecto de alfabetización, la idea de hacerse cargo del problema de la comunicación por una comunidad educativa. Si el audiovisual educa, Trabenco se ha puesto en juego para, a través de unos repertorios aprendidos, poner en juego sus disposiciones y cambiar el paradigma educomunicacional. El cambio de paradigma comunicacional también nos provoca una reflexión sobre un cambio en el paradigma educacional, ya que el espacio educativo debería acompañar a esta nueva realidad y desplegar una pedagogía para la vida. En algunos casos, en nuestra experiencia educativa, nos da la impresión de estar en una institución pedagógica del siglo XIX, con docentes del siglo XX y educandos del siglo XXI. Así, en los planteamientos de Freire (2010) para la pedagogía y de Kaplún (1998) para la educación comunicacional, descubrimos un aprendizaje de contexto donde las comunidades educativas deben generar sus propios fines y necesidades, modos de hacer autopoiéticos y autotélicos. El cambio de paradigma comunicacional debe ayudar, ser ejemplo, del cambio en la educación que se dará en este siglo, donde el esquema jerárquico se sustituye por relaciones dialógicas de intersubjetivad.

Dentro de los hallazgos que hemos encontrado en la investigación, nos ha parecido muy importante que las familias van aportando ideas e instrumentos al proyecto, lo cual no solo los hace partícipes, sino que forma parte de sus necesidades y de sus inteligencias. El audiovisual dispara diferentes niveles de análisis y cumple diferentes funciones, eso nos quedó claro con el ejemplo de la creación del plano de una chaqueta que se convirtió en tema de debate y en un microrrelato: En un grupo de autorreflexión decidieron tratar audiovisualmente cómo quedaba el centro a la salida. Hicieron grabaciones, pero una en concreto tuvo como protagonista a una chaqueta que nadie recogía y que todos los miembros de la comunidad educativa saltaban y esquivaban sin colocarla. Esto sirvió para una reflexión de toda la comunidad, pero inesperadamente una niña recogió el ejemplo y en una clase en la que estaban trabajando micro relatos realizó el siguiente:

"Estoy tumbada y sola en el suelo.

La gente me pisa, me da patadas, me esquivan, no me hacen ni caso.

Madres, padres, niños, hasta profesores.

Pero al fin alguien se da cuenta y me cuelga en el perchero.

¡iQué vida!!”. (Lúa, 9 años)

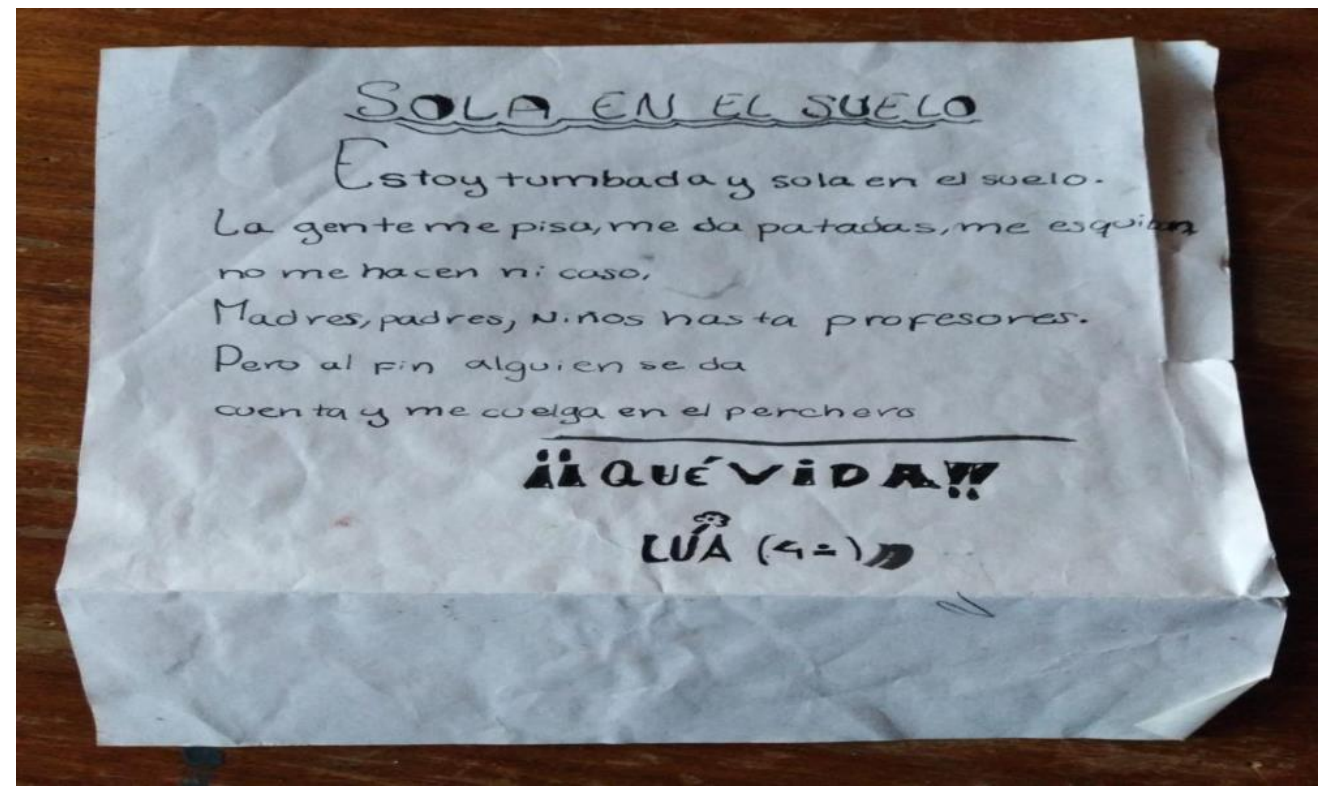

Figura 3. Microrrelato de una alumna de 4, microrrelato realizado dentro de la asignatura de Lengua a raíz de una práctica de alfabetización audiovisual en el colegio Trabenco; fotografiado del archivo del AMPA del colegio Trabenco (2019). 
Esto demuestra la fertilidad del audiovisual para la escuela, herramienta que necesita ser investigada para un uso más profundo y enriquecedor de las experiencias de aprendizaje.

Hemos descubierto que el audiovisual y sus prácticas de aprendizaje cambian los modos de relación familiares y de la comunidad educativa, con la consecuencia de cambiar los modos de ver de los participantes en el proyecto de alfabetización audiovisual. La actitud crítica sólo necesita el espacio para emerger, siendo el audiovisual una herramienta muy fértil para estas prácticas. Es también interesante destacar, con este ejemplo de acción pedagógica, la importancia de la eficiencia educativa de los proyectos colectivos y proyectos de la comunidad, siendo los acuerdos la base de la cultura democrática de la escuela y además una herramienta para el trabajo grupal de alfabetización audiovisual de la comunidad educativa. Nos ha parecido revelador la idea de la escuela como nuevo espacio de exhibición, viendo el centro educativo y las casas de los educandos como espacios naturales de materiales fílmicos compartidos. Además de la búsqueda de espacios de visionado colectivo alternativos a los cines de proyección mainstream. No nos gustaría olvidar la importancia que dan en este proyecto al trabajo en los cursos de infantil, el cual, tras su investigación, intenta empezar a introducir elementos de reflexión sobre este medio, ya que los niños y niñas de esta etapa son abordados por muchos más mensajes audiovisuales de los que en un principio podía parecer.

La potencia de este proyecto está en todos los elementos que confluyen en un modelo de escuela diferente, casi inexistente en el sistema público. Lo que creemos que hace posible que este proyecto cristalice en una experiencia concreta es, en primer lugar, que responde a un contexto de comunidad educativa, donde ésta se ha cohesionado fuertemente a través de un modo de relación asambleario, democrático y participativo. Al ser un colectivo pequeño, como colegio de línea 1, permite una mayor organización y solucionar problemas colectivamente. No es lo mismo organizar talleres para tres clases que para doce. El perfil de las familias (alto nivel de formación y compromiso con una educación alternativa a la tradicional), siendo un colegio público de una ciudad dormitorio, hace emerger un modo de relación dentro del centro que beneficia la participación. De este modo es más fácil llegar a un proyecto educativo que trabaja por proyectos y en el que no hay notas ni exámenes, lo cual facilita la posibilidad de hacer talleres. Al mismo tiempo, la cultura de acuerdos, pone en juego todos los mecanismos de participación para que los talleres se lleven a cabo, dentro de una clara cultura de escuela, donde la participación en ella forma parte de la vida cotidiana.

El proyecto ha tenido muy poca capacidad de conectividad y no ha sido exportado a otras comunidades educativas. Esto creemos que se debe a que es un proyecto de contexto con unas características muy concretas, basado en algo muy difícil de conseguir: una colectividad pequeña y un nivel de cohesión de la comunidad educativa que vemos muy pocas veces en la escuela pública, con lo cual creemos que no puede trasladarse la experiencia al completo, pero sí alguna de sus acciones pedagógicas que cada contexto educativo debe adaptar a sus posibilidades. pensamos que hay algo fundamental que puede exportarse a todo espacio educativo que pretenda una alfabetización audiovisual: no subestimar la infancia y no sobreestimar la tecnología. Las TIC están al alcance de la vida cotidiana y los educandos las manejan con naturalidad. Observando el proceso de los talleres, nos damos cuenta de que los 
educandos realizan vídeos y manejan aplicaciones de creación audiovisual sin problemas. Por otro lado, en ocasiones los docentes se sienten superados por las tecnologías, pero, como nos comenta por su experiencia Ana Benayas (profesora del primer ciclo de primaria de este centro), "realmente en cuanto los docentes empiezan a usar los dispositivos de grabación y reproducción audiovisual te das cuenta de que tienen una lógica de manejo muy intuitiva". Además, otro de los elementos fundamentales del triunfo de este proyecto es la sencillez, para ellos es más importante una película necesaria que perfecta. De este modo, esperamos haber aguzado la mirada como Marco Polo sobre las débiles luces de la alfabetización audiovisual.

\section{Referencias}

Aparici, Roberto y García Marín, David (2017) Comunicar y educar en el mundo que viene. Barelona: Gedisa.

Bernabeu Morón, N., Noemí, E. R., Laura, G. H., y Ana, R. P. (2011). Alfabetización mediática y competencias básicas. Madrid: Secretaría General Técnica. Subdirección General de Documentación y Publicaciones.

Bergala, Alain (2007) La hipótesis del cine. Barcelona: Laertes.

Berger, Jhon (2000). Modos de ver. Barcelona: Gustavo Gilli.

BOE (2012). Real Decreto-ley 14/2012, de 20 de abril, de medidas urgentes de racionalización del gasto público en el ámbito educativo.

Buckingham, David (2005) Educación en Medios, Barcelona: Paidós.

Calramonte, Jordi (2016). Estética modal. Madrid: Gedisa.

Calvino, Italo (2015). Las ciudades invisibles. Madrid: Siruela.

Cloutier, Jean (2001). Petit traité de communication.Montreal: Éditions Carte Blanche.

COPE (2013). "Wert anuncia la asignatura de cultura artística, visual y audiovisual".

Freire, Paulo (2010). Pedagogía de la indignación. Madrid: Morata.

Gabinete de Comunicación y Educación de la Universidad Autónoma de Barcelona (2015). Perspectivas 2015. El uso del audiovisual en las Aulas. Barcelona: Planeta. Dentro del Proyecto Filmed de la Comisión Europea 2015/0091.

INTEF (2017). Marco común de competencia digital docente. Octubre 2017

Kaplún, Mario (1998). Una pedagogía de la comunicación. Madrid: Ediciones de la Torre.
Ley Orgánica 8/2013, de 9 de diciembre, para la mejora de la calidad educativa (LOMCE). BOE, Madrid, España, 10 de diciembre de 2013.

Ley Orgánica 3/2020, de 29 de diciembre, por la que se modifica la Ley Orgánica 2/2006, de 3 de mayo, de Educación (LOMLOE). BOE, Madrid, España, 30 de diciembre de 2020

Hartmann, Nicolai (1956). Ontología. Mexico: FCE.

Lukács, György (1967). Estética. Barcelona- México. D.F.: Grijalbo.

MECD (2017). Notas de convenio de colaboración. El Ministerio de Educación, Cultura y Deporte y la Academia de Cine acuerdan crear el Portal Educativo del Cine Español. Diciembre, 4, 2017.

Orden ECD/65/2015, de 21 de enero, por la que se describen las relaciones entre las competencias, los contenidos y los criterios de evaluación de la educación primaria, la educación secundaria obligatoria y el bachillerato. BOE, Madrid, España, Sábado 29 de enero de 2015.

Orden ECD/2657/2015, de 1 de diciembre, por la que se conceden los premios Historia de la Cinematografía y Alfabetización Audiovisual para el curso 2014- 2015.

Real Decreto 126/2014, de 28 de febrero, por el que se establece el currículo básico de la Educación Primaria. BOE, Madrid, España, Sábado 1 de marzo de 2014.

Toffler, A. (1980). La tercera ola. Barcelona: Plaza y Janés.

Trabenco (2009). Proyecto para premio Marta Mata. Material de archivo consultado en el centro.

- (2010). Plan General Anual del curso 2010-2011. Material de archivo consultado en el centro.

- (2018). Proyecto "Educar la Mirada". Material de archivo consultado en el centro.

- (2021). http://www.trabenco.com/ [Consulta: 13 de julio de 2021 